\title{
A Continuous Dynamical Systems Approach to Gauss-Newton Minimization
}

\author{
Roy Danchick \\ 10336 Wilshire Boulevard, Unit 101, Los Angeles, USA \\ Email: dodeee@sbcglobal.net
}

Received 28 August 2014; revised 5 October 2014; accepted 9 November 2014

Copyright (C) 2014 by author and OALib.

This work is licensed under the Creative Commons Attribution International License (CC BY). http://creativecommons.org/licenses/by/4.0/

(c) (i) Open Access

\begin{abstract}
In this paper we show how the iterative Gauss-Newton method for minimizing a function can be reformulated as a solution to a continuous, autonomous dynamical system. We investigate the properties of the solutions to a one-parameter ODE initial value problem that involves the gradient and Hessian of the function. The equation incorporates an eigenvalue shift conditioner, which is a non-negative continuous function of the state. It enforces positive definiteness on a modified Hessian. Assuming the existence of a unique global minimum, the existence of a bounded connected sub-level set of the function and that the Hessian is non-zero in the interior of this set, our main results are: 1) existence of local solutions to the ODE initial value problem; 2) construction of a global solution by recursive extension of local solutions; 3 ) convergence of the global solution to the minimizing state for all initial values contained in the interior of the bounded level set; 4) eventual exact exponential decay of the gradient magnitude independent of the particular function and number of its variables. The results of a numerical experiment on the Rosenbrock Banana using a constant step-size 4th order Runge-Kutta method are presented and we point toward the direction of future research.
\end{abstract}

\section{Keywords}

Gauss-Newton Method, Unconstrained Minimization, Dynamical Systems, Ordinary Differential Equations

Subject Areas: Dynamical System, Numerical Mathematics, Ordinary Differential Equation

\section{Introduction}

This paper can be viewed as a generalization of the gradient method described in [1]. In that paper the authors derived sufficiency conditions for the solution, $x(t)$, of the ordinary differential equation initial value problem,

$$
x^{\prime}(t)=-\nabla f(x(t)), \quad x(0)=x_{0}
$$


to converge to the global minimum of $f$ as $t \rightarrow \infty$.

The motivation for our approach also arises from our previous paper [2] on the computation of accurate numerical partials. In [2] we constructed a minimization algorithm that exploited $O\left(h^{4}\right)$ accurate first and second order numerical partials. We investigated the convergence properties of the Gauss-Newton iterative method:

$$
\boldsymbol{x}_{i+1}=\boldsymbol{x}_{i}-\left(H\left(\boldsymbol{x}_{i}\right)+u\left(\boldsymbol{x}_{i}\right) I\right)^{-1} \nabla f\left(\boldsymbol{x}_{i}\right) \quad B=\left\{\boldsymbol{x}:\|\boldsymbol{x}\|_{2} \leq r\right\} \subset \mathbb{R}^{n}, \quad r \gg 1,
$$

to minimize the function $f: \mathbb{R}^{n} \rightarrow \mathbb{R}, n \geq 1, f \in C^{m}\left(\mathbb{R}^{n}\right), m \geq 4$ in which the gradient $\nabla f$ and Hessian $H$ were accurately approximated and $u \geq 0$ was an eigenvalue shift conditioner. It is computed to force $H+u I>0$ (positive definite) on each iteration. In this way the eigenvector structure of $H$ was preserved and under-relaxation was used in the algorithm, when required, to guarantee that $f$ was strictly decreasing from iteration to iteration. Moreover, the inclusion of the term $u\left(x_{i}\right)$ on the right hand side in (2) above was shown to be an effective valley-following technique.

In this paper we recast the discrete dynamical system above as a continuous dynamical system whose evolution is governed by the autonomous one-parameter ordinary differential equation:

$$
\begin{aligned}
& x^{\prime}(t)=-k(H(x(t))+u(x(t)) I)^{-1} \nabla f(x(t)), \quad x(0)=x_{0} \in D, \\
& D=\operatorname{int}\left(S\left(y_{0}\right)\right) .
\end{aligned}
$$

where, in addition to the smoothness conditions on $f$ above, the sub-level set $S\left(y_{0}\right)=\left\{\boldsymbol{y}: f(\boldsymbol{y}) \leq f\left(\boldsymbol{y}_{0}\right)\right\}$ is assumed to be bounded and connected.

The positive parameter $k$ is inserted into the right hand side (r.h.s.) of (3) above to increase the convergence rate. The eigenvalue shift conditioner $u$ is constructed so that the matrix $H(x(t))+u(x(t)) I$ stays positive definite for all $t \geq 0$. Here $I$ is the $n \times n$ identity matrix.

The main result in this paper is that if $\boldsymbol{x}^{*}$ minimizes $f(\boldsymbol{y}), \boldsymbol{y} \in D$, such that $\boldsymbol{x}^{*}$ is the unique solution in $D$ to $\nabla f(\boldsymbol{y})=0, H(\boldsymbol{y}) \neq 0$ in $D, H\left(\boldsymbol{x}^{*}\right)>0$, and the sub-level set $S\left(\boldsymbol{y}_{0}\right)=\left\{\boldsymbol{y}: f(\boldsymbol{y}) \leq f\left(\boldsymbol{y}_{0}\right)\right\}$ is bounded and connected, then for each initial value $x_{0} \in D$ there is a global solution, $x(t)$, to (3), such that, for all $t \in[0, \infty), \boldsymbol{x}(t) \in D$ and $\lim _{t \rightarrow \infty} \boldsymbol{x}(t)=\boldsymbol{x}^{*}$; i.e. that the system (3) is asymptotically stable in the Lyapunov sense in $D$. Moreover, we show that there exists $T>0$ such that for all $t \geq T$

$$
\|\nabla f(x(t))\|_{2} /\|\nabla f(x(T))\|_{2}=\exp (-k(t-T))
$$

independent of the function $f$ and the dimension $n$.

The remainder of this paper is divided into 6 sections. Section 2 provides the notation we will use throughout. Section 3 is a short set of definitions. Section 4 is devoted to proofs. Section 5 discusses our results, conclusions, and directions for future work. Section 6 lists our references.

Throughout the paper we use the Euclidian norm induced topology so that the distance between $y \in \mathbb{R}^{n}$ and $\mathbf{z} \in \mathbb{R}^{n}$ is $\|\boldsymbol{y}-\mathbf{z}\|_{2}=\left[(\boldsymbol{y}-\mathbf{z})^{\mathrm{T}}(\boldsymbol{y}-\mathbf{z})\right]^{1 / 2}$ where the superscript $T$ means vector or matrix transposition.

\section{Notation}

$$
\boldsymbol{y}=\left(y_{1}, y_{2}, \cdots, y_{n}\right)^{\mathrm{T}} \in \mathbb{R}^{n} .
$$

$f \in C^{m}\left(\mathbb{R}^{n}\right)$ is the $m$ times continuously differentiable function $(m \geq 4)$ to be minimized $\nabla \boldsymbol{f}(\boldsymbol{y})=\left(\partial f / \partial y_{1}, \partial f / \partial y_{2}, \cdots, \partial f / \partial y_{n}\right)^{\mathrm{T}}$ is the gradient of $f$ at an arbitrary point $\boldsymbol{y} \in \mathbb{R}^{n}$.

$H(\boldsymbol{y})=\left(\partial^{2} f / \partial y_{i} \partial y_{j}\right)_{1 \leq i, j \leq n}$ is the Hessian matrix of $f$ at $\boldsymbol{y}$.

$\Lambda=\left(\lambda_{1}(\boldsymbol{y}), \lambda_{2}(\boldsymbol{y}), \cdots, \lambda_{n}(\boldsymbol{y})\right)$ are the $n$ real eigenvalues of $H(\boldsymbol{y})$.

\section{Definitions}

$S\left(\boldsymbol{y}_{0}\right)=\left\{\boldsymbol{y}: f(\boldsymbol{y}) \leq f\left(\boldsymbol{y}_{0}\right)\right\}$ is a bounded, connected sub-level set. 
$\boldsymbol{x}^{*}=\underset{\boldsymbol{y} \in S\left(\boldsymbol{y}_{0}\right)}{\arg \operatorname{minf}}(\boldsymbol{y})$ is unique such that $\nabla f\left(\boldsymbol{x}^{*}\right)=0, H\left(\boldsymbol{x}^{*}\right)>0$ and for all $\boldsymbol{y} \in S\left(\boldsymbol{y}_{0}\right) \boldsymbol{y} \neq \boldsymbol{x}^{*}$ implies $f(y)>f\left(x^{*}\right)$.

$D=\operatorname{int} S\left(y_{0}\right)$ is the interior of $S\left(y_{0}\right)$.

$B=\partial S\left(y_{0}\right)=S\left(y_{0}\right) \backslash D=\left\{\boldsymbol{y}: f(\boldsymbol{y})=f\left(\boldsymbol{y}_{0}\right)\right\}$ is the boundary of $S\left(\boldsymbol{y}_{0}\right)$.

$\mathrm{v}(\boldsymbol{y})=-k \nabla \boldsymbol{f}^{\mathrm{T}}(\boldsymbol{y})[H(\boldsymbol{y})+u(\boldsymbol{y}) I]^{-1} \nabla \boldsymbol{f}(\boldsymbol{y})$.

$\rho(\boldsymbol{y}, B)$ is the distance from $\boldsymbol{y}$ to $B$ for an arbitrary point $\boldsymbol{y} \in S\left(\boldsymbol{y}_{0}\right)$.

\section{Derivations}

\subsection{Compactness of $S\left(y_{0}\right)$}

We first assume that there exists a $\boldsymbol{y}_{0} \in \mathbb{R}^{n}, \quad \boldsymbol{y}_{0} \neq \boldsymbol{x}^{*}$, such that the sub-level set $S\left(\boldsymbol{y}_{0}\right)$ is bounded and connected. From the uniqueness of $\boldsymbol{x}^{*}$ it follows that $f\left(y_{0}\right)>f\left(\boldsymbol{x}^{*}\right)$. Since $S\left(y_{0}\right)$ is closed it follows that $S\left(y_{0}\right)$ is compact. Since $f\left(y_{0}\right)-f\left(x^{*}\right)>0$ the continuity of $f$ implies that there exists an open hyperspherical neighborhood of $\boldsymbol{x}^{*}, U=\left\{\boldsymbol{y}:\left\|\boldsymbol{y}-\boldsymbol{x}^{*}\right\|_{2}<\delta\right\}$, such that $\boldsymbol{y} \in U$ implies

$f(\boldsymbol{y})-f\left(\boldsymbol{x}^{*}\right)<f\left(\boldsymbol{y}_{0}\right)-f\left(\boldsymbol{x}^{*}\right)$ which implies $f(\boldsymbol{y})-f\left(\boldsymbol{y}_{0}\right)<0$. This last inequality implies $U \subset D$. So $D$ is a non-trivial connected open set, containing $x^{*}$, with compact closure $S\left(y_{0}\right)$.

\subsection{The Existence of Local Solutions}

For arbitrary $\boldsymbol{x}_{0} \in D$ such that $\boldsymbol{x}_{0} \neq \boldsymbol{x}^{*}$, we begin by partitioning the eigenvalues of $H\left(\boldsymbol{x}_{0}\right)$ : $\left(\lambda_{1}\left(x_{0}\right), \lambda_{2}\left(x_{0}\right), \cdots, \lambda_{n}\left(x_{0}\right)\right)$, into three disjoint sets.

$$
\Lambda^{-}=\left\{i: \lambda_{i}<0\right\}, \quad \Lambda^{0}=\left\{i: \lambda_{i}=0\right\}, \quad \Lambda^{+}=\left\{i: \lambda_{i}>0\right\} .
$$

Let

$$
\begin{aligned}
& u\left(x_{0}\right)=\max \left\{-2 \lambda_{i}: i \in \Lambda^{-}\right\} \text {if } \Lambda^{-} \text {is not empty, } \\
& u\left(x_{0}\right)=0 \text { if } \Lambda^{-} \cup \Lambda^{0} \text { is empty, } \\
& u\left(x_{0}\right)=\min \left\{\lambda_{i}: i \in \Lambda^{+}\right\} \text {if } \Lambda^{-} \text {is empty and } \Lambda^{0} \text { is not empty. }
\end{aligned}
$$

The assumption that $H \neq 0$ in $D$ and (5) and (6) implies that $H\left(x_{0}\right)+u\left(x_{0}\right) I>0$. Since the eigenvalues of $H\left(x_{0}\right)$ are continuous functions of the $n(n+1) / 2$ different entries of $H\left(x_{0}\right)$ and these are, in turn, continuous functions of $x_{0}$, each eigenvalue, $\lambda_{i}, i=1,2, \cdots, n$, is a continuous function of $x_{0}$. It then follows that $u\left(x_{0}\right)$ is also a continuous function of $\boldsymbol{x}_{0}$. The continuity of $u$ at $\boldsymbol{x}_{0}$ implies that each of the elements of the matrix $\left(H\left(x_{0}\right)+u\left(x_{0}\right) I\right)^{-1}$ is continuous at $x_{0}$ as is each component of the matrix-vector product $\left(H\left(x_{0}\right)+u\left(x_{0}\right) I\right)^{-1} \nabla f\left(x_{0}\right)$.

Now let $M=k\left(\sup _{\boldsymbol{y} \in \boldsymbol{S}\left(\boldsymbol{y}_{0}\right)}\left\|[H(\boldsymbol{y})+u(\boldsymbol{y})]^{-1} \nabla f(\boldsymbol{y})\right\|_{2}\right)$ and $b_{1}=\rho\left(\boldsymbol{x}_{0}, B\right) / 2$.

Following Ganesh in [3], let

$$
t_{1}=\min \left(b_{1} /(k M), 1\right)>0 .
$$

By the Peano existence theorem for ordinary differential equations, there exists a local solution to (3), $\boldsymbol{x}_{1}(t)$, in the interval $I_{1}=\left[0, t_{1}\right]$, which will be confined to the compact hyper-spherical neighborhood $\left\{\boldsymbol{y}:\left\|\boldsymbol{y}-\boldsymbol{x}_{0}\right\|_{2} \leq b_{1}\right\} \subset D$. This follows because any such local solution to (3) satisfies

$$
\boldsymbol{x}_{1}(t)=\boldsymbol{x}_{0}-k \int_{0}^{t}\left[H\left(\boldsymbol{x}_{1}(s)\right)+u\left(\boldsymbol{x}_{1}(s)\right) I\right]^{-1} \nabla f\left(\boldsymbol{x}_{1}(s)\right) \mathrm{d} s .
$$

From (8) we then have for all $t \in I_{1}$ :

$$
\left\|\boldsymbol{x}_{1}(t)-\boldsymbol{x}_{0}\right\|_{2} \leq k \int_{0}^{t}\left[H\left(\boldsymbol{x}_{1}(s)\right)+u\left(\boldsymbol{x}_{1}(s)\right) I\right]^{-1} \nabla f\left(\boldsymbol{x}_{1}(s)\right) \mathrm{d} s \leq \int_{0}^{t} M \mathrm{~d} s=M t \leq b_{1} .
$$


Thus, for all $t \in I_{1}, \quad x_{1}(t)$ is a smooth curve and

$$
f^{\prime \prime}\left(x_{1}(t)\right)=v\left(x_{1}(t)\right) \text {. }
$$

That is, $f\left(x_{1}(t)\right)$ is a smooth non-increasing function for all $t \in I_{1}$.

Also note that

$$
f\left(x_{1}\left(t_{1}\right)\right)<f\left(y_{0}\right)
$$

Since $x_{1}\left(t_{1}\right) \in D$.

\subsection{Extension of Local Solutions and the Existence of a Global Solution}

Let $\boldsymbol{w}(0)=x_{1}\left(t_{1}\right)$. Using the same construction as above there is a positive number $b_{2}$ such that (3) has a smooth solution, $w(t)$, for all $t \in\left[0, t_{2}\right]$, where $t_{2}$ is computed from (7) with $b_{2}$ substituting for $b_{1}$. If we now define:

$$
\begin{aligned}
& \boldsymbol{x}_{2}(t)=\boldsymbol{x}_{1}(t) \text { for } t \in\left[0, t_{1}\right), \\
& \boldsymbol{x}_{2}(t)=\boldsymbol{w}\left(t-t_{1}\right) \text { for } t \in\left[t_{1}, t_{1}+t_{2}\right)
\end{aligned}
$$

we can extend the smooth local solution $x_{1}(t), t \in\left[0, t_{1}\right]$, to the smooth solution $x_{2}(t), t \in I_{2}=\left[0, t_{1}+t_{2}\right]$.

Letting

$$
T_{L}=\sum_{l=1}^{L} t_{l},
$$

it is clear, from the construction above, that assuming $x_{L}(t) \in D$ for all $t \in I_{L}=\left[0, T_{L}\right]$ we can extend $\boldsymbol{x}_{L}(t)$, $t \in I_{L}$ to $x_{L+1}(t), t \in I_{L+1}$ by the same argument as above. Thus we have an inductive proof on $L$ that there exists a global solution, $x(t)$, to (3) in $\left[0 \sup _{L \rightarrow \infty} T_{L}\right)$ such that for each $L=1,2, \cdots, t \in I_{L}$ implies

$\boldsymbol{x}(t)=\boldsymbol{x}_{L}(t)$ with (10) holding in this same interval. It should also be noted that (10) holds for each $\boldsymbol{x}(t)$, $t \in\left[0, \sup _{L \rightarrow \infty} T_{L}\right)$. We now prove the following lemma:

\section{Lemma.}

$$
\sup _{L \rightarrow \infty} T_{L}=\infty .
$$

\section{Proof.}

Suppose (14) is false. Then $b_{l} \rightarrow 0$ as $l \rightarrow \infty, \quad \boldsymbol{x}\left(t_{l}\right) \rightarrow y_{\partial} \in B$, and for each $\varepsilon>0$ there exists $L$ such that, for all $l>L, f\left(y_{\partial}\right)-f\left(x\left(t_{l}\right)\right)=f\left(y_{0}\right)-f\left(x\left(t_{l}\right)\right)<\varepsilon$. If we choose $\varepsilon=f\left(y_{0}\right)-f\left(x_{0}\right)$ then, for all sufficiently large $l$.

$$
f\left(y_{0}\right)-f\left(x\left(t_{l}\right)\right)<f\left(y_{0}\right)-f\left(x_{0}\right) \text { or } f\left(x_{0}\right)<f\left(x\left(t_{l}\right)\right),
$$

contradicting the non-increasing property of $f(x(t))$. So (14) is true, $f(x(t))$ is non-increasing and:

$$
\lim _{t \rightarrow \infty} f(x(t))=\lim _{L \rightarrow \infty} f\left(x\left(T_{L}\right)\right)=f_{\text {lim }}>-\infty .
$$

\subsection{Asymptotic Stability of the Dynamical System (3) in D}

The proof below closely follows the treatment of Lyapunov stability by Boyd in [4].

Suppose that $f_{\lim }>f\left(\boldsymbol{x}^{*}\right)$, consider the sequence $\left\{\boldsymbol{x}\left(T_{L}\right)\right\}_{L=1}^{\infty}$. Since $S\left(\boldsymbol{y}_{0}\right)$ is compact, there is a convergent subsequence $\left\{\boldsymbol{x}\left(T_{L_{r}}\right)\right\}_{r=1}^{\infty}$ such that

$$
\lim _{r \rightarrow \infty} \boldsymbol{x}\left(T_{L_{r}}\right)=\boldsymbol{x}_{\lim } \in S\left(y_{0}\right), \quad \lim _{r \rightarrow \infty} f\left(\boldsymbol{x}\left(T_{L_{r}}\right)\right)=f\left(\boldsymbol{x}_{\lim }\right)=f_{\text {lim }} .
$$


From (17) it follows that $\lim _{t \rightarrow \infty} \boldsymbol{x}(t)=\boldsymbol{x}_{\lim } \in S\left(\boldsymbol{y}_{0}\right)$ exists and, for all $t \in[0, \infty)$, $f\left(\boldsymbol{x}_{\lim }\right) \leq f(\boldsymbol{x}(t)) \leq f(\boldsymbol{x}(0))$. Therefore $C=\left\{\boldsymbol{y}: f\left(\boldsymbol{x}_{\lim }\right) \leq f((\boldsymbol{y})) \leq f(\boldsymbol{x}(0))\right\}$ is compact, $\boldsymbol{x}^{*} \notin C$, and

$$
\sup _{\boldsymbol{y} \in C} v(\boldsymbol{y})=-a<0 \text {. }
$$

It then follows that

$$
f(x(T))=f(x(0))+\int_{0}^{T} v(x(s)) d s \leq f(x(0))-a T .
$$

Taking limits on both sides of the inequality (19) above:

$$
\lim _{T \rightarrow \infty} f(x(T)) \leq \lim _{T \rightarrow \infty}[f(x(0))-a T]=-\infty
$$

contradicting (16). It can thus be concluded that $f_{\lim }=f\left(x_{\lim }\right) \leq f\left(\boldsymbol{x}^{*}\right)$, which implies that $\boldsymbol{x}_{\lim }=\boldsymbol{x}^{*}$ and $\nabla f\left(x^{*}\right)=0$. Since $x_{0} \in D$ was arbitrary we can conclude that the system (3) is asymptotically stable in $D$ in the sense of Lyapunov stability.

\subsection{Eventual Exponential Gradient Decay}

Given the sufficiency conditions in Section 1, there is an open hyper-spherical neighborhood $U^{+}=\left\{\boldsymbol{y} \in S\left(\boldsymbol{y}_{0}\right):\left\|\boldsymbol{y}-\boldsymbol{x}^{*}\right\|_{2}<\rho\right\}$ such that, for all $\boldsymbol{y} \in \bar{U}^{+}$, the positive eigenvalues of $H(\boldsymbol{y})$ are bounded away from zero. That is, $f$ is convex in $y \in \bar{U}^{+}$. Thus, recalling (6), for all $y \in U^{+}, u(y)=0$. Since $\lim _{t \rightarrow \infty} \boldsymbol{x}(t)=\boldsymbol{x}^{*}$ there exists $T$ such that for all $t>T \quad \boldsymbol{x}(t) \in U^{+}$. Therefore, for all $t>T$, (3) reduces to:

$$
\boldsymbol{x}^{\prime}(t)=-k(H(\boldsymbol{x}(t)))^{-1} \nabla f(\boldsymbol{x}(t)) .
$$

Since

$$
H(x(t))(x(t))^{\prime}=(\nabla f(x(t)))^{\prime},
$$

pre-multiplying both sides of (21) above by $H(x(t))$ we obtain, for all $t>T$,

$$
(\nabla f(x(t)))^{\prime}=-k \nabla f(x(t))
$$

From the continuity of $\nabla f$ along $x(t)$ the gradient boundary value at $t=T$ is $\nabla f(x(T))$. Thus the solution to (23) is, for all $t \geq T$

$$
\nabla f(x(t))=\exp (-k(t-T)) \nabla f(x(T)) .
$$

From (24) above it follows that, for all $t \geq T$

$$
\|\nabla f(x(t))\|_{2} /\|\nabla f(x(T))\|_{2}=\exp (-k(t-T))
$$

independent of the function $f$ and the dimension $n$. This implies eventual exponential decay for all functions $f \in C^{4}\left(\mathbb{R}^{n}\right)$ that satisfy the sufficiency conditions assumed in our proof.

\subsection{Eventual Sub-Level Set Convexity}

The eventual sub-level set convexity property states that for all $y \in U^{+}$the sub-level sets $S(\boldsymbol{y})=\{\mathbf{z}: f(\mathbf{z}) \leq f(\boldsymbol{y})\}$ are convex. This follows easily from the convexity of $f$ in $U^{+}$as proved below:

Suppose $\boldsymbol{z}, \boldsymbol{w} \in S(\boldsymbol{y})$ and $\lambda \in[0,1]$. The convexity of $f$ in $U^{+}$implies

$$
f(\lambda \mathbf{z}+(1-\lambda) \boldsymbol{w}) \leq \lambda f(\mathbf{z})+(1-\lambda) f(\boldsymbol{w}) \leq \lambda f(\boldsymbol{y})+(1-\lambda) f(\boldsymbol{y})=f(\boldsymbol{y}) .
$$

Thus $\lambda \mathbf{z}+(1-\lambda) \boldsymbol{w} \in S(\boldsymbol{y})$ as was to be shown. 


\subsection{The Sub-Level Set Inclusion Property}

The sub-level set inclusion property states that for all open hyper-spherical neighborhoods $U$ of $\boldsymbol{x}^{*}$, such $U \subset \bar{U}^{+}$there is a sub-level set, $S(\mathbf{z}), \quad \mathbf{z} \neq \boldsymbol{x}_{0}$, such that $S(\mathbf{z}) \subset U$.

Proof.

Let $\left\{\boldsymbol{y}_{n}\right\}, \boldsymbol{y}_{n} \in \bar{U}^{+}, n \in Z^{+}, \boldsymbol{y}_{n} \rightarrow \boldsymbol{x}^{*}$. We make the following definitions:

$$
S_{n}=S\left(\boldsymbol{y}_{n}\right), d_{n}=\operatorname{diam}\left(S_{n}\right)=\sup \left(\|\boldsymbol{w}-\mathbf{z}\|_{2}, \boldsymbol{w}, \mathbf{z} \in S_{n}\right)=\left\|\boldsymbol{w}_{n}-\mathbf{z}_{n}\right\|_{2}, \quad \boldsymbol{w}_{n}, \mathbf{z}_{n} \in \partial\left(S_{n}\right) .
$$

From the above it follows that

$$
f\left(\boldsymbol{w}_{n}\right)=f\left(\mathbf{z}_{n}\right)=f\left(\boldsymbol{y}_{n}\right) .
$$

We prove that $\boldsymbol{w}_{n} \rightarrow \boldsymbol{x}^{*}$. Suppose not. Then there is a $\delta>0$ and an infinite set $N_{\delta} \subset Z^{+}$such that, for all $n \in N_{\delta},\left\|\boldsymbol{w}_{n}-\boldsymbol{x}^{*}\right\|_{2} \geq \delta$ Consider the compact set $S_{\varepsilon}=\left\{\boldsymbol{y} \in \bar{U}^{+}:\left\|\boldsymbol{y}-\boldsymbol{x}^{*}\right\|_{2} \geq \varepsilon\right\}$ and let $\varepsilon=\inf \left\{f(y): y \in S_{\delta}\right\}-f\left(x^{*}\right)>0$. Then, for all $n \in N_{\varepsilon}, f\left(y_{n}\right)-f\left(x^{*}\right) \geq \varepsilon$, contradicting $y_{n} \rightarrow x^{*}$. The same argument goes through to show that $\mathbf{z}_{n} \rightarrow \boldsymbol{x}^{*}$. Thus $\left\|\boldsymbol{w}_{n}-\mathbf{z}_{n}\right\|=d_{n} \rightarrow 0$ and there is an $n$ such that $d_{n}<\operatorname{diam}(U) / 2$. It follows that, for all $\boldsymbol{w} \in S_{n},\left\|\boldsymbol{w}-\boldsymbol{x}^{*}\right\|_{2} \leq d_{n}<\operatorname{diam}(U) / 2$. Thus $S_{n} \subset U$ as was to be shown.

\section{Results, Conclusions and Future Directions}

\subsection{Results}

In summary, given the reasonably weak sufficiency conditions assumed in Section 1 above, we have shown that $\left\{x^{*}\right\}$ is a global attractor in $D$ for the asymptotically stable system (3) and that, after a finite time, the magnitude of the gradient decays exactly exponentially, independent of the function $f$ and the dimension $n$. The practical implication of these results are that the convergence, $\boldsymbol{x}(t) \rightarrow \boldsymbol{x}^{*}$ as $t \rightarrow \infty$, occurs independent of the starting point $\boldsymbol{x}_{0} \in D$. Moreover, for sufficiently large $t$, the solution is unique because the mapping $\phi$ : $U^{+} \rightarrow \nabla f\left(U^{+}\right), \quad z=\nabla f(y), \partial z / \partial y=H(y)>0$ is a diffeomorphism with inverse $\phi^{-1}$ such that, for all $t \geq T$.

$$
\phi^{-1}(\nabla f(x(t)))=\phi^{-1}(\exp (t-T) \nabla f(x(T)))=x(t) .
$$

Thus, for $t \geq T$ the solution depends uniquely on the gradient boundary value $\nabla f(x(T))$. With the convexity and inclusion properties in hand it is easy to infer that, for sufficiently large $t, \boldsymbol{x}(t) \rightarrow \boldsymbol{x}^{*}$, evolves through a family of convex sub-level sets whose diameters shrink to zero as $t \rightarrow \infty$. It is also noteworthy that, while $f$ is monotone, it does not follow that $\|\nabla f\|_{2}$ is monotone as well. This is the case because:

$$
\left(\|\nabla f\|_{2}^{2}\right)^{\prime}=-2 k\|\nabla f\|^{\mathrm{T}} H(H+u I)^{-1}\|\nabla f\|_{2} .
$$

The eigenvalues of $H(H+u I)^{-1}$ are $\lambda_{i} /\left(u+\lambda_{i}\right), i=1,2, \cdots, n$. Thus, if $u>0$, it cannot be guaranteed that the time derivative of the gradient magnitude squared be always negative as is the case with the function $f$ itself.

The initial value $\boldsymbol{x}_{0} \in D$ to start a numerical solution to (3) is easily found by taking a sufficiently small step from $\boldsymbol{y}_{0}$ in the direction $-\nabla f\left(y_{0}\right)$ into $D$. In practice, it will always be possible to start at $\boldsymbol{y}_{0}$ because of the continuity of the solution and because the solution will always move from $y_{0}$ into $D$.

\section{Examples}

1) The Rosenbrock function

The "Rosenbrock Banana" $f: \mathbb{R}^{2} \rightarrow \mathbb{R}, f=\left(1-x_{1}\right)^{2}+100\left(x_{2}-x_{1}^{2}\right)^{2}$ with parameter $c=100$ shown in Figure 1(a) is a well-known test function, designed to challenge the effectiveness of a candidate minimization algorithm such as the one described in [2]. As can be seen in Figure 1(a) its graph features a long, narrow valley, along the line $x_{1}-x_{2}=0$, containing the minimum. This valley must be followed closely by the sequence 


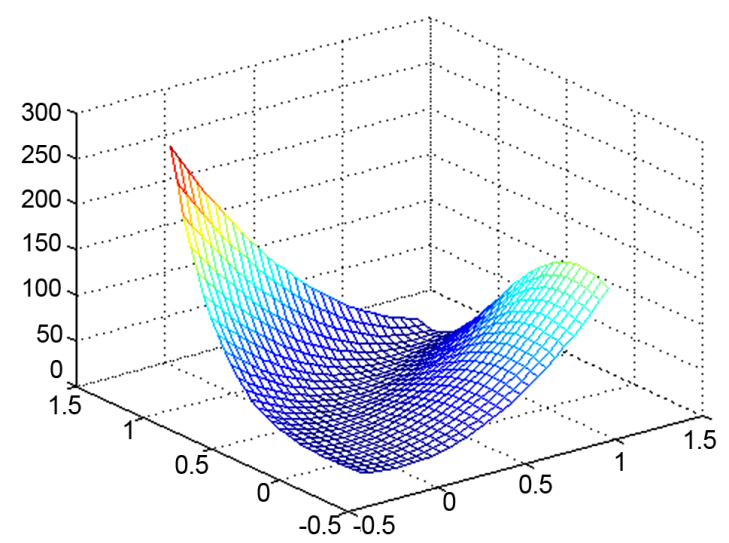

(a)

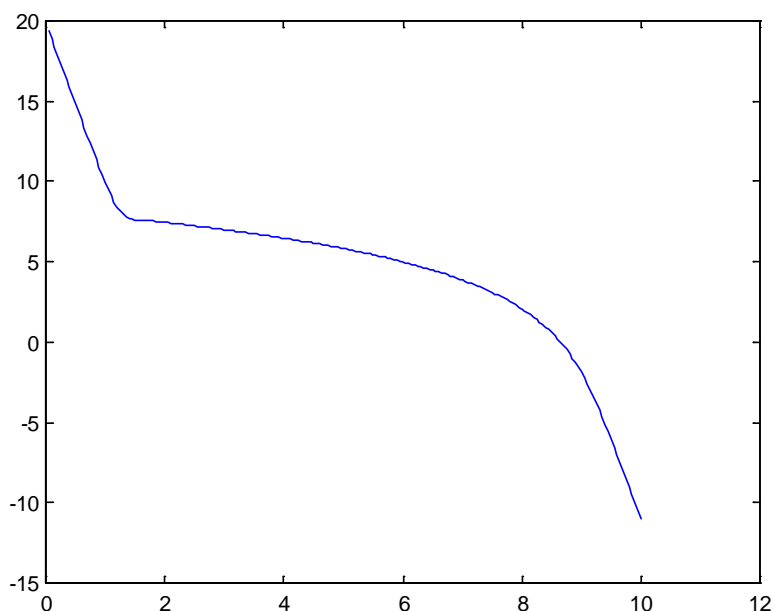

(b)

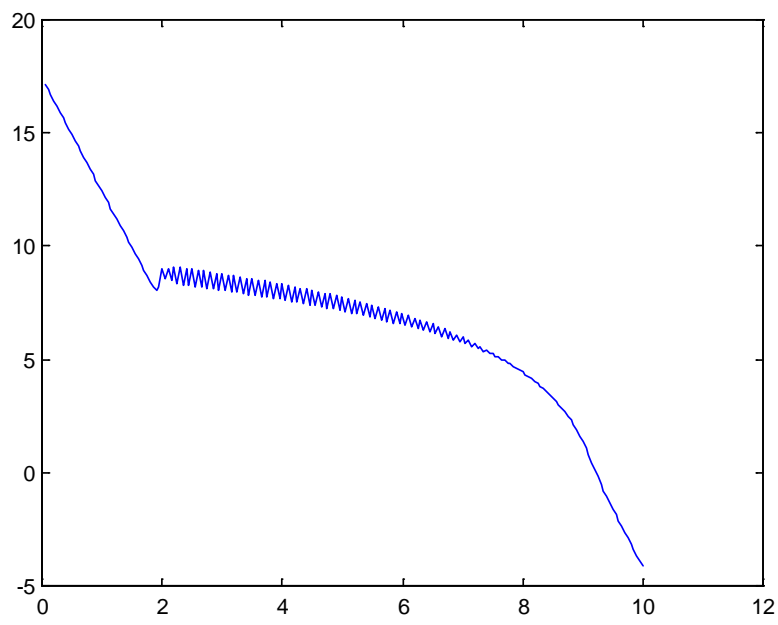

(d)

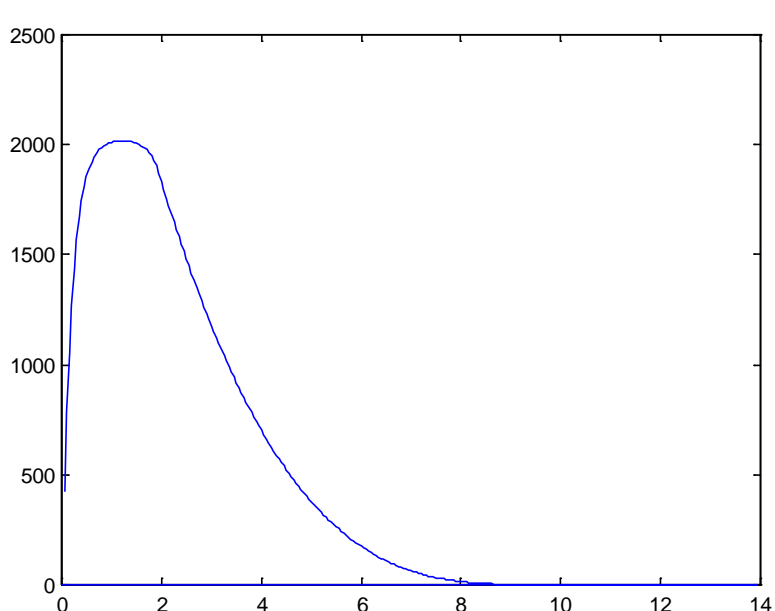

(c)

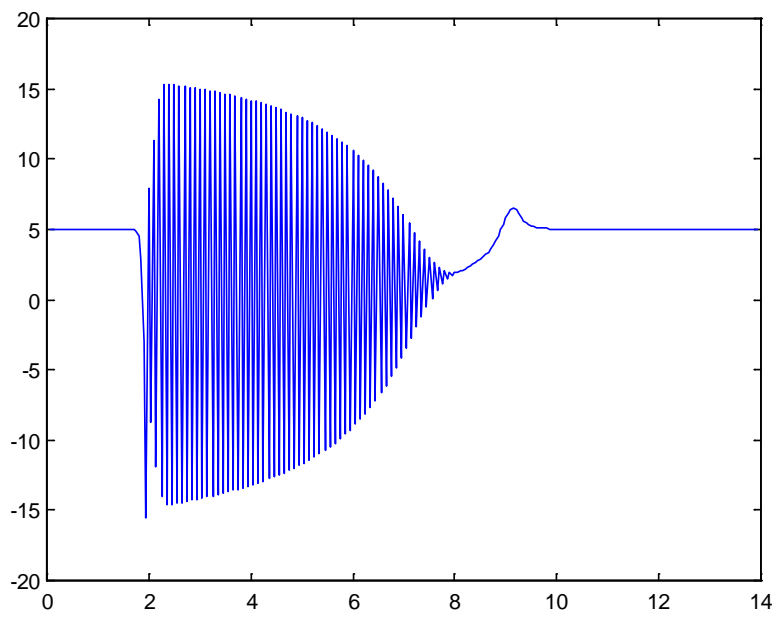

(e)

Figure 1. (a) The Rosenbrock Banana; (b) $\ln (|f(x(t))|)$ vs. time; (c) Distance from $x(t)$ to $\boldsymbol{x}^{*}$ vs. time; (d) $\ln \left(\|\nabla f(x(t))\|_{2}\right) \quad$ vs. time; (e) $-\left(\ln \left(\|\nabla f((n+1) h)\|_{2} /\|\nabla f(n h)\|_{2}\right) / h \quad\right.$ vs. time.

of iterates toward the minimum of zero at $\boldsymbol{x}^{*}=[1,1]^{\mathrm{T}}$, or else the sequence will diverge. It was the valleyfollowing success of the discrete iterative algorithm [2] from a variety of starting guesses that inspired the writing of this paper. 
Figure 1(b) is a graph of the Euclidian distance between a MATLAB fourth order, constant step-length $h$ (0.05) Runge-Kutta method numerical solution to (3) and $\boldsymbol{x}^{*}$. A uniformly distributed random starting guess $\boldsymbol{x}_{0}=[45.01292851471754,-26.88614864257122]^{\mathrm{T}}$ was drawn in the rectangle: $-50 \leq x_{1} \leq 50,-50 \leq x_{2} \leq 50$. It should be noted that all of the Rosenbrock Banana's sub-level sets are bounded. So, because of uniqueness of the minimum, we should indeed expect convergence from any starting point in the rectangle. Figure 1 (c) is a graph of the function $\ln (|f(x(t))|)$ along the numerical solution and shows rapid convergence to zero. Figure 1(d) graphs $\ln \left(\|\nabla f(x(t))\|_{2}\right)$. This graph is consistent with theory in showing that $\ln \|\nabla f(x(t))\|_{2}$ is not monotone decreasing but does eventually decrease as $k t$, consistent with theory. Figure $1(\mathrm{e})$ plots the RungeKutta method's generated table of values of $-[\ln (\nabla f((n+1) h) / \nabla f(n h))] / h$ vs. time. The results that we have derived here say that the value should eventuatly reach and remain at constant value $k=5$. This, up to numerical integration error, can be seen to be the case for all time beyond approximately 9.5.

2) The ellipsoidal function

The function of three real variables, whose sub-level set boundaries are long, thin ellipsoids:

$f: \mathbb{R}^{3} \rightarrow R, f(x)=100\left(x_{1}-0.1\right)^{2}+\left(x_{2}-1\right)^{2}+0.1\left(x_{3}-100\right)^{2}$, provides the second example. Because of its unique global minimum of zero at $\boldsymbol{x}=[0.1,1,100]^{\mathrm{T}}$, vanishing gradient, and constant positive define Hessian it perfectly displays, from the start, the exact exponential gradient decay independent of dimension that is our fundamental result. Figure 2(a) is a graph of the function on the plane $x_{3}=100$. Figure 2(b) is a graph of the Euclidian distance in $\mathbb{R}^{3}$ from a point on the curve at time $t$ to the minimizing point starting at time $t=0$ with initial conditions $\boldsymbol{x}_{0}=[50,-50,50]^{\mathrm{T}}$. Figure 2(c) shows the anticipated expected constant exponential decrease in the value of the function along the trajectory, while Figure 2(d) shows the constant exponential decay in gradient magnitude over a time interval from zero to sec. that our theory predicts. Finally, Figure 2(e) shows the expected constancy of $-[\ln (\nabla f((n+1) h) / \nabla f(n h))] / h$ along the numerically integrated trajectory. In this case we kept $k=5$ but reduce used $h$ to 0.1 .

\subsection{Conclusions}

There are four basic concepts that provide the foundation for our results: 1) eigenvalue shift conditioning; 2) the Peano existence theorem; 3) Lyapunov stability theory; and 4) the notion of sub-level sets.

Eigenvalue shift conditioning of the Hessian matrix enables the construction of a quasi Lyapunov function$V(t)=f(\boldsymbol{x}(0))+\int_{0}^{t} v(\boldsymbol{x}(s)) \mathrm{d} s$ - that has all the properties of a classical Lyapunov function except for the nonessential minimal function value of zero at the origin of $\mathbb{R}^{n}$.

The Peano existence theorem underlies the construction of local solutions that can be concatenated to construct a global solution.

The concept of sub-level sets, especially in the light of their eventual convexity and inclusion properties, is a useful, natural setting to study the properties of the solution to the dynamical system (3).

Moreover our results show why the concept of eigenvalue shift conditioning is so effective in [2]. Informally one could view the iterative algorithm constructed there as a crude Euler numerical integration of the dynamical system (3) in which the step-size was 1 with an occasional decrease to the fractional under-relaxation parameter to some integral power $\geq 1$. The key idea was to keep the function values decreasing from iteration-to-iteration. This decrease was guaranteed by eigenvalue shift conditioning. In fact it appears at first glance to be relatively easy to revisit the theory underlying the algorithm and mimic the approach in this paper to prove that, given the same assumptions of this paper, the iterations always converge to the unique minimum.

The drawback to solving the minimization problem by the numerical integration of the dynamical system (3) is, of course, that each integration step requires multiple numerical determinations of the eigenvalues and eigenvectors of the Hessian (four such evaluations in the case of a fourth order Runge-Kutta method). This is numerically expensive especially when contrasted to the performance of the discrete algorithm, 200 such determinations for the continuous vs. 20 for the discrete. The results derived in [5] which halve the number of derivative evaluations from four to two per step down for a fourth order Runge-Kutta method is one approach to increase efficiency. Another approach is to exploit a reliable variable step numerical method such as the one developed by this author and co-author in [6]. This method, which has a fixed global accuracy order of six but with variable step-size also requires just two derivative evaluations per step. The catch is that $f$ may not be sufficiently 


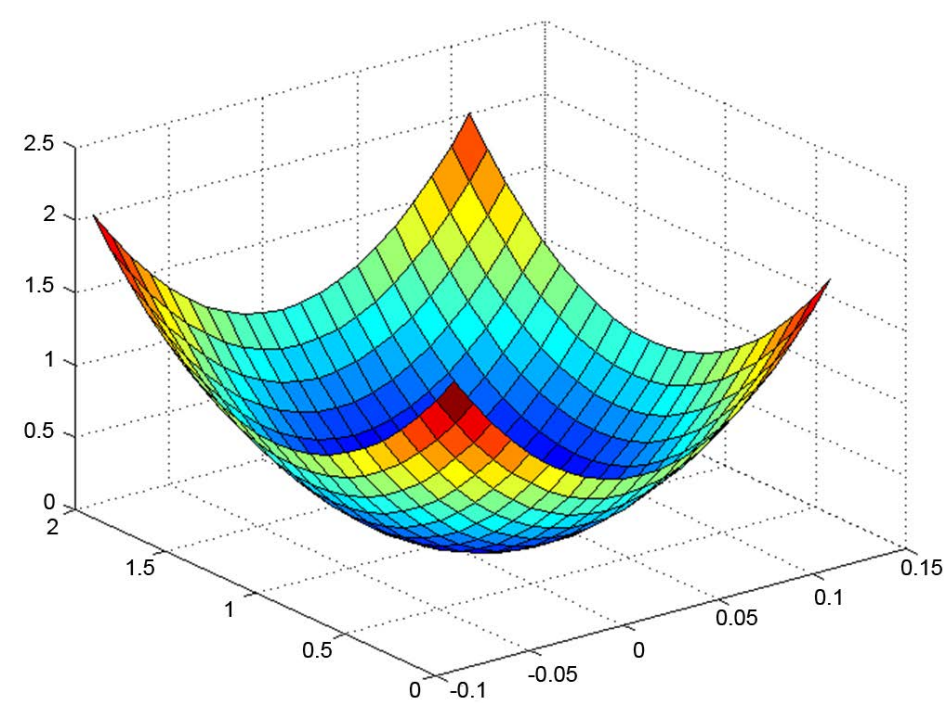

(a)

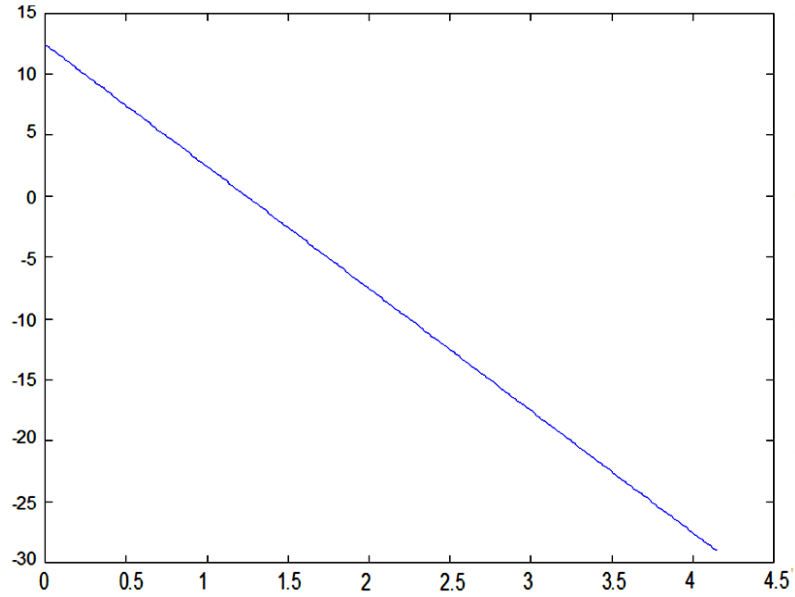

(b)

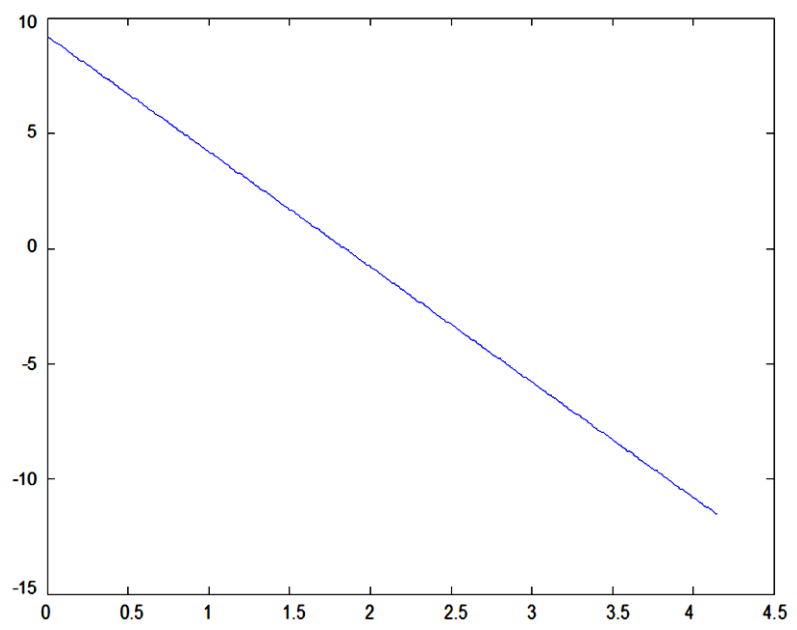

(d)

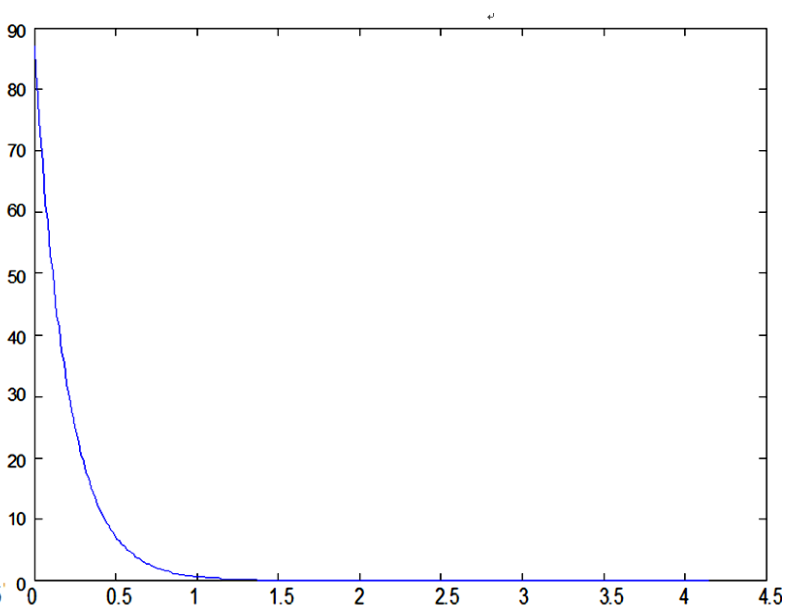

(c)

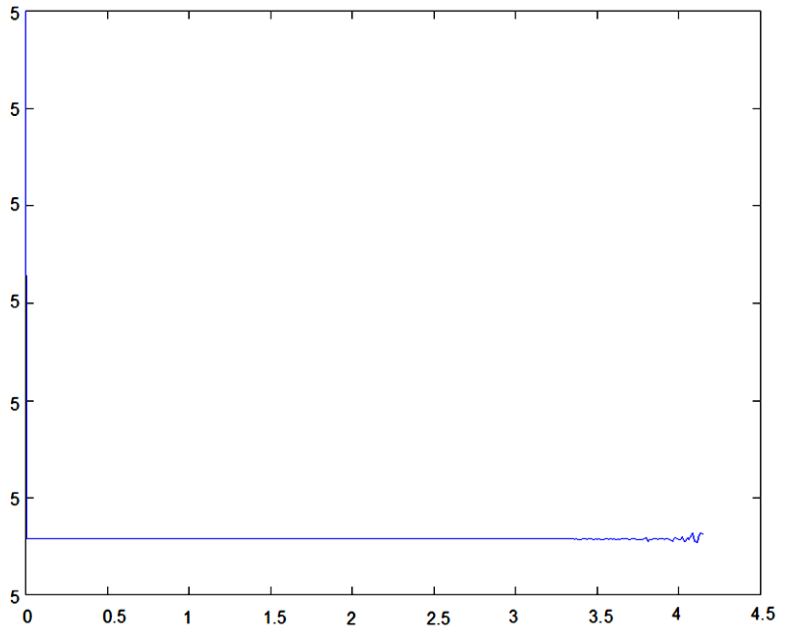

(e)

Figure 2. (a) Section of the ellipsoidal function; (b) $\ln (|f(x(t))|)$ vs. time; (c) Distance from $\quad x(t)$ to $\boldsymbol{x}^{*}$ vs. time; (d) $\ln \left(\|\nabla f(\boldsymbol{x}(\boldsymbol{t}))\|_{2}\right) \quad$ vs. time; (e) $-\left(\ln \left(\|\nabla f((n+1) h)\|_{2} /\|\nabla f(n h)\|_{2}\right) / h \quad\right.$ vs. time. 
differentiable to take full advantage of the method's high order of global accuracy.

A second area ripe for investigation is the potential for mating the dynamical systems approach developed here with the accurate numerical partials method of [2] to approximate gradient and Hessian. This approach will avoid the tedious and error-prone computation of first and second partials for problems of very large dimension with complicated first and second partials. Of course, the issue of computational efficiency might be problematic because each gradient/Hessian numerical approximation requires $2 n^{2}+n+1$ function evaluations.

Finally, with our results for unconstrained minimization established, the next natural step would be to investigate the extension of our method to extend our approach to the case of non-unique vanishing of the gradient and the general nonlinear programming problem.

\section{References}

[1] Liao, L.-Z., Qi, L.Q. and Tam, H.W. (2005) A Gradient-Based Continuous Method for Large-Scale Optimization Problems. Journal of Global Optimization, 31, 271-286. http://dx.doi.org/10.1007/s10898-004-5700-1

[2] Danchick, R. (2006) Accurate Numerical Partials with Applications to Optimization. Applied Mathematics and Computation, 183, 551-559. http://dx.doi.org/10.1016/j.amc.2006.05.083

[3] Ganesh, S.S. (2007) Lecture Notes on Ordinary Differential Equations. Annual Foundation School, IIT, Mumbai, 3-28 December.

[4] Boyd, S. (2008-2009) Lecture 12 Basic Lyapunov Theory, Electrical Engineering 363 Course Notes, Stanford University, Stanford, 10.

[5] Someijer, B.P. (1986) On the Economization of Explicit Runge-Kutta Methods. Applied Mathematics and Computation, 2, 57-69.

[6] Danchick, R. and Juncosa, M. (2006) Maximum Polynomial Degree Nordsieck-Gear (k, p) Methods: Existence, Stability, Consistency, Refinement, Convergence, and Computational Examples. Applied Mathematics and Computation, 182, 907-933. http://dx.doi.org/10.1016/j.amc.2006.04.067 